\title{
TRANSVERSUS ABDOMINIS PLANE (TAP) BLOCK AS A MODALITY FOR POSTOPERATIVE ANALGESIA IN ABDOMINAL SURGERIES
}

\author{
Shikha Sharma1 ${ }^{1}$ Nandita Mehta ${ }^{2}$, Asim Khan ${ }^{3}$
}

${ }^{1}$ Assistant Professor, Department of Anaesthesiology and Intensive Care, Acharya Shri Chander College of Medical Sciences and Hospital, Jammu.

${ }^{2}$ Professor and HOD, Department of Anaesthesiology and Intensive Care, Acharya Shri Chander College of Medical Sciences and Hospital, Jammu.

${ }^{3}$ Resident, Department of Anaesthesiology and Intensive Care, Acharya Shri Chander College of Medical Sciences and Hospital, Jammu.

ABSTRACT
BACKGROUND
Transversus Abdominis Plane (TAP) block is a regional analgesic technique which provides analgesia after abdominal surgery.
There is a sensory blockade of abdominal wall skin and muscles via local anaesthetic deposition above transversus abdominis
muscle. We evaluated the efficacy of the TAP block in the first 24 hours after abdominal surgery (Midline or non-midline) in a
prospective, randomised controlled study.

\section{MATERIALS AND METHODS}

Sixty adult patients undergoing elective abdominal surgery under general anaesthesia were included in this study and were randomly assigned to TAP block group or control group. After induction of anaesthesia, unilateral or bilateral TAP block (depending upon site of incision) was performed using $20 \mathrm{~mL}$ of $0.5 \%$ Ropivacaine on each side. Intraoperatively, haemodynamic parameters like $\mathrm{HR}, \mathrm{SBP}, \mathrm{DBP}, \mathrm{MBP}$ and $\mathrm{SPO}_{2}$ till the end of surgery. Postoperatively, patients were assessed for pain with VAS score for 24 hours at regular intervals by a blinded investigator.

\section{RESULTS}

TAP block significantly reduced the pain scores, requirement for rescue and demand analgesia in the unilateral and bilateral study groups. Postoperative VAS scores (both at rest and on coughing) in the TAP block group were significantly lower than those in the control group. The respiratory rate, intraoperative and postoperative pulse rate were also significantly lower in the TAP block group.

\section{CONCLUSION}

TAP block using $0.5 \%$ ropivacaine provided longer duration of analgesia than that in the control group in patients undergoing abdominal surgeries. TAP block via lumbar approach provides better pain relief especially in major lower abdominal surgeries as compared to upper abdominal surgeries. No complications attributable to TAP block or drug under study were detected.

\section{KEYWORDS}

Transversus Abdominis Plane Block, Neurofascial Plane, Petit Triangle, Ropivacaine.

HOW TO CITE THIS ARTICLE: Sharma S, Mehta N, Khan A. Transversus abdominis plane (TAP) block as a modality for postoperative analgesia in abdominal surgeries. J. Evolution Med. Dent. Sci. 2017;6(43):3385-3389, DOI: $10.14260 /$ Jemds $/ 2017 / 733$

\section{BACKGROUND}

Pain remains the most dreaded fear of humans associated with any type of surgery. A major contribution to the pain experienced by a patient after an abdominal surgery is from the incision made in the abdominal wall. ${ }^{1}$ Even a relatively minor operation such as inguinal herniorrhaphy may lead to chronic pain in about $12 \%$ of patients, which has a clinically significant effect on daily activities if not adequately taken care of. ${ }^{2}$ Various modalities have been used to manage postoperative pain like non-steroidal anti-inflammatory

Financial or Other, Competing Interest: None.

Submission 25-04-2017, Peer Review 19-05-2017,

Acceptance 22-05-2017, Published 29-05-2017.

Corresponding Author:

Dr. Shikha Sharma,

\#41, Vikas Lane-1,

Talab Tillo, Jammu,

Jammu \& Kashmir.

E-mail: shikha_2527@yahoo.co.in

DOI: $10.14260 /$ jemds $/ 2017 / 733$ drugs (NSAIDS), opioids and epidural block, each of which has its own side-effects. The opioids have number of side effects such as respiratory depression, emesis, reduction in motility of gut and sedation. Use of NSAIDs may lead to altered haemostasis, renal dysfunction, and gastrointestinal haemorrhage. Peripheral nerve blockade is an alternative means of providing analgesia, by anaesthetizing the sensory nerves.

Transversus abdominis plane (TAP) block is gaining popularity as one of such regional blocks. TAP block can be performed through the lumbar triangle of Petit formed by external oblique muscle anteriorly, latissimus dorsi muscle posteriorly, iliac crest inferiorly and is usually identified as a defect $1 \mathrm{~cm}$ above the iliac crest in the midaxillary line. ${ }^{2}$ The technique involves injection of local anaesthetic into the plane between the transversus abdominis and internal oblique muscles. It allows sensory blockade of plexus of nerves supplying abdominal wall skin and muscles via local anaesthetic drug deposition above the transversus abdominis muscle. Over time, local anaesthetic and the concentration of local anaesthetic and the concentration of local anaesthetic 
used for TAP block has changed. Bupivacaine when used in TAP block provides longer duration of action but has been shown to have selective cardiac effects due to the slow rate at which it dissociates from the sodium channel. Ropivacaine is a relatively new amino amide local anaesthetic and exists as an S-enantiomer, with low systemic toxicity than bupivacaine.3,4 Although some studies indicate higher concentration of ropivacaine is needed as compared to bupivacaine; but it has been established that ropivacaine $0.5 \%$ is equi-effective as bupivacaine $0.25 \%$. The present study was planned to compare the postoperative analgesic efficacy of TAP block using ropivacaine $0.5 \%$ unilaterally or bilaterally in patients undergoing abdominal surgery.

\section{MATERIALS AND METHODS}

This study protocol of this randomised controlled trial was approved by the Institutional Ethics Committee. Written informed consent was obtained from all patients prior to their enrolment. Patients with American Society of Anaesthesiologists (ASA) physical status I - II scheduled for elective abdominal surgery were randomised to receive TAP block in addition to standardised anaesthesia care or standardised anaesthesia care only.

A convenience sample of sixty patients was enrolled for this study ( $\mathrm{n}=30$ for both groups). A between-group difference of 2 in VAS scores was considered to be clinically significant.

The inclusion criteria were ASA I and II patients, aged 16 to 65 years, of either sex, with BMI $18-30 \mathrm{~kg} / \mathrm{m}^{2}$. The exclusion criteria were: patient refusal to procedure; history of allergy to local anaesthetics; patients with infection at the site of injection; inherited or acquired coagulopathy; patients on systemic anticoagulation therapy; patient who had received analgesic drug 24 hours prior to induction of anaesthesia; and patients with ASA Grade III and IV. Subjects who fulfilled the inclusion criteria were randomly assigned to either of the two groups according to computer generated table of randomisation. Group A or block group $(n=30)$ : Patients in this group received $20 \mathrm{~mL} 0.5 \%$ ropivacaine in TAP block (unilateral for unilateral incision/bilateral for midline or transverse incisions) and Group B or control group $(n=30)$ : Patients in this group received standardised anaesthesia care and did not receive any TAP block.

All patients underwent pre-anaesthetic check-up on the day immediately prior to surgery and were administered alprazolam $0.5 \mathrm{mg}$, pantoprazole $40 \mathrm{mg}$ and domperidone 10 mg via the oral route at bedtime. The patients were told to report pain in the postoperative period using the visual analogue scale (VAS $0=$ no pain and VAS $10=$ worst possible pain). In operation theatre after attaching the monitors, baseline values of non-invasive blood pressure (NIBP), pulse oximetry (SpO2), end tidal carbon dioxide (EtCO2) and electrocardiography (ECG) were noted. Prior to induction of anaesthesia, parenteral ranitidine $(50 \mathrm{mg})$ and Tramadol (1 $\mathrm{mg} / \mathrm{kg}$ ) were administered. Anaesthesia was induced with Inj. Propofol ( $2 \mathrm{mg} / \mathrm{kg}$ ) and Inj. Rocuronium $(0.6 \mathrm{mg} / \mathrm{kg})$ and an appropriate-sized cuffed endotracheal tube was placed. Immediately after intubation, TAP block was performed using $20 \mathrm{~mL}$ of $0.5 \%$ Ropivacaine in the block group before surgical incision and no block in the control group (unilaterally or bilaterally depending on the type of incision). Inj. Ondansetron $(0.1 \mathrm{mg} / \mathrm{kg})$ was administered intraoperatively.
Anaesthesia was maintained with oxygen (33\%), nitrous oxide $(66 \%)$ and isoflurane of varying concentrations.

\section{Technique of Block}

In this study, the classic landmark 'two-pop technique' was used to administer TAP block, as described by McDonnell and co-workers in 2007.5 After optimal positioning with the patient supine, the target area was properly draped under all aseptic precautions. A $90 \mathrm{~mm} 22 \mathrm{G}$ bevelled spinal needle (Quincke) was inserted perpendicular to skin, just superior to iliac crest $(2-3 \mathrm{~cm})$ within lumbar triangle of Petit and slightly behind the midaxillary line. Gentle advancement of needle resulted in two pop sensations; one as needle entered the plane between the external oblique and internal oblique fascial layers, and the second as it entered the plane between the internal oblique and the transversus abdominis muscle, i.e., the target site. Following correct position of the needle and repeated negative aspiration, $20 \mathrm{~mL}$ of $0.5 \%$ ropivacaine with epinephrine $(0.1 \mathrm{mg})$ as intravascular marker was injected in $5 \mathrm{~mL}$ increments. In case of midline incisions, a bilateral TAP block was performed. Patients in whom the heart rate increased by $20-30$ beats $/ \mathrm{min}$. from baseline within first $60 \mathrm{sec}$. were excluded from the study and were monitored and managed accordingly.

Intraoperatively, haemodynamic parameters like blood pressure (systolic, diastolic and mean), heart rate and oxygen saturation were recorded at $0,5,10,20$ minutes (min.) and every 10 minutes thereafter till the end of surgery. At the end of surgery, patient was reversed with Inj. Neostigmine 0.05 $\mathrm{mg} / \mathrm{kg})$ and Inj. Glycopyrrolate $(0.01 \quad \mathrm{mg} / \mathrm{kg})$ Postoperatively, the presence and severity of pain was assessed systematically using Visual Analogue Scale (VAS) at $0,2,4,8,12,18$ and 24 hours (hrs.). The VAS score $(0=$ no pain; 10=most severe pain) was recorded at rest and coughing. Any patient with a VAS score of more than 3 qualified for rescue analgesia in form of Inj. Diclofenac 1.5 $\mathrm{mg} / \mathrm{kg} \mathrm{IV}$ up to a maximum of three doses over a 24-hour period; in case of inadequate analgesia, patients were administered inj. Tramadol $1 \mathrm{mg} / \mathrm{kg}$ IV in $50 \mathrm{~mL}$ normal saline infusion, titrated to a VAS score of no more than 5 , with maximum of three doses over 24 hours. At the end of 24 hours, all patients were evaluated with respect to pain score, quality of analgesia and total analgesic requirement (total number of injections in a group; average number of injections per patient and the total number of patients who required analgesia. Any side effects complained by the patients were recorded postoperatively and managed appropriately.

Between-group differences with respect to parametric variables were assessed with independent sample $t$ test or Chi-Squared test; those with respect to non-parametric variables were assessed by Mann-Whitney test. Betweengroup differences associated with $\mathrm{P}$ values $<0.05$ were considered statistically significant.

\section{RESULTS}

Data pertaining to baseline characteristics and key intraoperative and postoperative parameters are presented in Table 1. Both groups were comparable with respect to age, sex, ASA physical status grade. Intraoperative haemodynamic parameters such as SBP, DBP, and MBP remained stable and were comparable in both the groups; however, intra operative heart rate was significantly higher in the control 
group. Mean post-operative heart rate in the control group was significantly higher than that in the block group (Table 2).

VAS scores in the Block group, both at rest and on coughing, at $0,2,4,8,12,18$ and 24 hrs. were significantly higher than those in the Control group (Tables 3, 4).

The number of patients requiring analgesia, the mean time to first demand analgesia and total analgesic requirement in the control group was significantly higher than that in the block group. In the TAP block group, postoperative pain control was better in patients who underwent lower abdominal surgery (such as that for inguinal hernia) as compared to those who underwent upper abdominal surgery (such as open cholecystectomy) in the block group. Although, the difference in VAS scores was statistically significant, the absolute difference was less than 1 at all time-points. This is a study limitation.

\begin{tabular}{|c|c|c|c|}
\hline Variable & Block Group & Control Group & P value \\
\hline Age & $48.9 \pm 16.8$ & $48.2 \pm 16.1$ & $.844^{*}$ \\
\hline $\operatorname{Sex}(M / F) \%$ & $36.67 / 63.33$ & $30 / 70$ & $0.317^{* *}$ \\
\hline Duration of surgery (Min.) & $90.8 \pm 16.4$ & $89.6 \pm 11.4$ & 0.699 \\
\hline Intraoperative heart rate (per min.) & $75.4 \pm 2.3$ & $90.4 \pm 1.9$ & $0.001^{* *}$ \\
\hline $\begin{array}{c}\text { Intraoperative BP (mmHg) } \\
\text { Systolic } \\
\text { Diastolic } \\
\text { Mean }\end{array}$ & $\begin{array}{c}133.7 \pm 6.6 \\
81.7 \pm 3.2 \\
96.9 \pm 4.2\end{array}$ & $\begin{array}{c}135.8 \pm 5.6 \\
84.4 \pm 2.9 \\
98.7 \pm 3.4\end{array}$ & $\begin{array}{l}0.435^{* *} \\
0.342^{* *} \\
0.900^{* *}\end{array}$ \\
\hline Postoperative heart rate (first 24 hours) & $76.7 \pm 3.1$ & $89.9 \pm 3.5$ & $0.001^{* *}$ \\
\hline $\begin{array}{c}\text { Postoperative BP (first 24 hours) } \\
\text { Systolic } \\
\text { Diastolic } \\
\text { Mean }\end{array}$ & $\begin{array}{c}131.7 \pm 6.6 \\
77.7 \pm 3.2 \\
96.6 \pm 4.2\end{array}$ & $\begin{array}{c}132.8 \pm 5.6 \\
78.4 \pm 2.9 \\
96.7 \pm 3.4\end{array}$ & $\begin{array}{l}0.435^{* *} \\
0.342^{* *} \\
0.900^{* *}\end{array}$ \\
\hline $\begin{array}{c}\text { VAS score } \\
\text { At rest } \\
\text { During coughing }\end{array}$ & $\begin{array}{l}2.9 \pm 0.3 \\
3.1 \pm 0.3\end{array}$ & $\begin{array}{l}3.5 \pm 0.3 \\
3.8 \pm 0.3\end{array}$ & $\begin{array}{l}0.001^{\text {** }} \\
0.001^{\text {** }}\end{array}$ \\
\hline Time to first demand analgesia (Hours) & 8.5 & 4 & $0.012^{* *}$ \\
\hline Total analgesic requirement (no. of Inj.) (Rescue/Demand) & $35 / 13$ & $40 / 34$ & $0.001^{*}$ \\
\hline Mean Nausea score & $0.84 \pm 0.52$ & $1.67 \pm 0.62$ & $0.001^{* *}$ \\
\hline Mean postoperative respiratory rate (per min.) & $14.9 \pm 0.7$ & $17.1 \pm 1.1$ & $0.001^{* *}$ \\
\hline
\end{tabular}

* P value calculated by Chi-Squared test.

** $\mathrm{P}$ value calculated by independent sample t test.

Data presented as mean ( \pm standard deviation).

\begin{tabular}{|c|c|c|c|}
\hline Postop HR & Block Group & Control Group & P value* $^{*}$ \\
\hline PACU & $77.7 \pm 5.7$ & $90.5 \pm 2.7$ & 0.001 \\
\hline 2 hrs. & $76.2 \pm 7.1$ & $90.8 \pm 6.0$ & 0.001 \\
\hline 4 hrs. & $80.5 \pm 7.4$ & $89.0 \pm 6.9$ & 0.001 \\
\hline 8 hrs. & $78.2 \pm 7.7$ & $89.6 \pm 6.7$ & 0.001 \\
\hline 12 hrs. & $75.8 \pm 6.4$ & $90.9 \pm 6.0$ & 0.001 \\
\hline 18 hrs. & $75.9 \pm 7.1$ & $90.7 \pm 7.1$ & 0.001 \\
\hline 24 hrs. & $72.8 \pm 4.4$ & $87.7 \pm 4.9$ & 0.001 \\
\hline \multicolumn{4}{|c|}{ Table 2. Postoperative Heart Rate by Study Group } \\
\hline
\end{tabular}

Data presented as mean ( \pm standard deviation).

*Between-group difference is statistically significant at all time-points (independent sample $t$ test).

\begin{tabular}{|c|c|c|c|}
\hline VAS (Rest) & Block Group & Control Group & P value \\
\hline VAS PACU & $3.0 \pm 0.6$ & $3.9 \pm 0.8$ & $0.001^{*}$ \\
\hline VAS 2 hrs. & $2.9 \pm 0.6$ & $3.5 \pm 0.8$ & $0.001^{*}$ \\
\hline VAS 4 hrs. & $3.2 \pm 0.7$ & $3.5 \pm 0.9$ & 0.088 \\
\hline VAS 8 hrs. & $2.9 \pm 0.6$ & $3.5 \pm 0.9$ & $0.002^{*}$ \\
\hline VAS 12 hrs. & $2.9 \pm 0.7$ & $3.5 \pm 0.8$ & $0.001^{*}$ \\
\hline VAS 18 hrs. & $2.6 \pm 0.6$ & $3.5 \pm 0.8$ & $0.001^{*}$ \\
\hline VAS 24 hrs. & $2.5 \pm 0.3$ & $3.1 \pm 0.7$ & $0.001^{*}$ \\
\hline \multicolumn{4}{|r|}{ Table 3. VAS Scores at Rest at Various Time-points } \\
\hline
\end{tabular}

Data presented as mean ( \pm standard deviation).
* Between-group difference is statistically significant at all time-points (independent sample $t$ test).

\begin{tabular}{|c|c|c|c|}
\hline VAS (Cough) & Block Group & Control Group & P value* \\
\hline VAS PACU & $3.2 \pm 0.6$ & $4.3 \pm 0.8$ & 0.001 \\
\hline VAS 2 hrs. & $3.2 \pm 0.7$ & $3.8 \pm 0.8$ & 0.001 \\
\hline VAS 4 hrs. & $3.4 \pm 0.7$ & $3.8 \pm 0.9$ & 0.040 \\
\hline VAS 8 hrs. & $3.2 \pm 0.6$ & $3.8 \pm 0.9$ & 0.002 \\
\hline VAS 12 hrs. & $3.1 \pm 0.7$ & $3.8 \pm 0.8$ & 0.001 \\
\hline VAS 18 hrs. & $2.9 \pm 0.6$ & $3.8 \pm 0.8$ & 0.001 \\
\hline VAS 24 hrs. & $2.7 \pm 0.3$ & $3.4 \pm 0.8$ & 0.001 \\
\hline
\end{tabular}

Table 4. VAS Scores on Coughing at Various Time-points

Data presented as mean ( \pm standard deviation).

* Between-group difference is statistically significant at all time-points (independent sample t test).

\section{DISCUSSION}

Adequate postoperative analgesia confers several advantages; it reduces the stress response, and helps achieve better surgical outcomes. Effective pain control also facilitates rehabilitation and accelerates postoperative recovery. Other benefits of effective regional analgesic techniques include reduced pain intensity, decreased incidence of side effects from analgesics, and improved patient comfort. Abdominal field blocks have been recognised 
as very effective in achieving significant postoperative analgesia in patients undergoing abdominal surgical procedures such as open cholecystectomy, inguinal herniorrhaphy, laparotomy and caesarean delivery. ${ }^{6}$

However, the lack of clearly defined anatomical landmarks has meant that the full potential of abdominal wall blockade in patients undergoing major abdominal procedures remains to be realised. The classical two-pop technique as described by McDonnell in 2007 was used in this study, as it is a simple technique and does not require sophisticated equipment. However, direct visualisation of anatomic structures by ultrasound and deposition of drug under vision is more advantageous. The use of ultrasonography is associated with substantially increased costs and skilled personnel. In setups where ultrasonography aided technique is not an option, the blind landmark technique is a good option owing to its relative simplicity and efficacy. ${ }^{7}$ The TAP provides a space into which local anaesthetic can be deposited to achieve myocutaneous sensory blockade.8,9,10 Deposition of the local anaesthetic dorsal to the mid-axillary line also blocks the lateral cutaneous afferents, thus facilitating blockade of the entire anterior abdominal wall. The lumbar triangle of Petit is an easily identifiable, fixed, and palpable landmark, and is located dorsal to the mid-axillary line. ${ }^{11}$ The transversus abdominis neurofascial plane can easily be accessed via this triangle, and local anaesthetic is deposited into this plane, using the loss of resistance technique. ${ }^{12}$

In this randomised, prospective clinical trial, TAP block was administered using $20 \mathrm{~mL}$ of $0.5 \%$ ropivacaine on each side, which corresponded to a dose of $100 \mathrm{mg}$ on each side. McDonnell et al (2008) in their study found that the maximum recommended dose for TAP block is $150 \mathrm{mg}$ on each side. ${ }^{6}$ The TAP block in this study proved that dose of ropivacaine $100 \mathrm{mg}$ is effective in providing analgesia in the postoperative period and there was no reported case of any systemic toxicity. Our results are in line with Fauladi et al who evaluated efficacy of unilateral TAP block with bupivacaine and ropivacaine for postoperative analgesia in patients undergoing lower abdominal surgeries such as hernia repair and appendicectomy. ${ }^{13}$ They concluded that $0.5 \%$ ropivacaine provided longer duration of analgesia than $0.25 \%$ bupivacaine when used for TAP block in patients undergoing lower abdominal surgery. In this study, TAP block reduced postoperative pain scores in the study group as compared to that in the control group, both at rest and on coughing, during the first 24 postoperative hours. Similar results were reported by Laffey et al in 2008 who administered bilateral blocks in patients undergoing large bowel resection. 5 In our study, TAP block significantly reduced the demand for rescue and demand analgesia in the TAP block group as assessed by the average no. of analgesics required per patient. The total number of injections for rescue and demand analgesia was also higher in control group. These findings are consistent with results reported by Patil et al in 2010, who found benefits of TAP block in patients undergoing laparotomy. ${ }^{14}$

The vitals were monitored postoperatively at regular intervals for first $24 \mathrm{hrs}$. in this study. The average heart rate in the TAP block group was lower than that in the control group, which possibly suggests controlled sympathetic stress response secondary to adequate pain relief in patients receiving TAP block. However, blood pressure readings did not show any statistically significant difference between the two groups. No major complications attributable to the TAP block were observed. An isolated case of transient femoral nerve paraesthesia was encountered in a female patient that resolved on its own within 6-7 hrs. postoperatively.

The limitations of this study were that the postoperative pain was studied for 24 hours only and patient satisfaction score was not observed as far as postoperative analgesia was concerned. Also, ultrasound was not used in this study which is definitely a better option; however, it comes with extra cost of the needles and apparatus.

Thus, TAP block can do away with common side effects associated with opioids, respiratory depression being the most common one. Although epidural analgesia is superior to that with systemic opioids and provides excellent extended analgesia in the postoperative period covering both visceral and somatic pain. However, haemodynamic instability following the cardiovascular effects of epidural block is avoided with TAP block. Furthermore, TAP block can preserve bladder and lower limb motor function, thereby assisting early mobilisation after surgery. TAP block can provide unilateral analgesia, a potential advantage in patients undergoing non-midline incision. Although, the difference in VAS scores was statistically significant, the absolute difference was less than 1 at all time-points. This was the limitation of this study and future studies with larger sample size and with standardisation of the types of abdominal surgery included should be planned to draw more definitive conclusions.

\section{CONCLUSION}

TAP block is an emerging technique that significantly reduces the pain scores postoperatively and helps maintain intraoperative haemodynamic stability in patients undergoing midline and non-midline abdominal surgeries. It provides an excellent option for unilateral analgesia. 0.5\% Ropivacaine can be used routinely for TAP block for abdominal surgeries for effective analgesia. This block holds considerable promise as it reduces postoperative pain effectively; the benefit is particularly more evident in the case of lower abdominal surgeries. The two-pop technique is easy to perform and can be used in settings where facility for ultrasound is not available in the operation theatres.

\section{REFERENCES}

[1] Wall PD, Melzack R. Pain Measurements in Persons in Pain. In: Wall PD, Melzack R, editors. Textbook of Pain. 4th ed. Edinburgh UK: Churchill Livingstone; 1999. pp. 409-426.

[2] Aasvang E, Kehlet H. Chronic postoperative pain: the case of inguinal herniorrhaphy. $\mathrm{Br} \mathrm{J}$ Anaesth 2005;95(1):69-76.

[3] Kuppuvelumani P, Jaradi H, Delilkan A. Abdominal nerve blockade for postoperative analgesia after caesarean section. Asia Oceania J Obstet Gynaecol. 1993;19:165-9. 
[4] Dierking GW, Dahl JB, Kanstrup J, Dahl A, Kehlet H. Effect of pre-vs postoperative inguinal field block on postoperative pain after herniorrhaphy. Br J Anaesth. 1992;68:344-8.

[5] McDonnell J, Laffey J. Transversus abdominis plane block. Anesthesia and analgesia. 2007;105:883

[6] McDonnell JG, Curley G, Carney J, et al. The analgesic efficacy of transversus abdominis plane block after caesarean delivery: a randomized controlled trial. Anesth Analg 2008;106(1):186-91.

[7] Kawahara R, Tamai Y, Yamasaki K, et al. The analgesic efficacy of ultrasound-guided transversus abdominis plane block with mid-axillary approach after gynaecologic laparoscopic surgery: a randomized controlled trial. J Anaesthesiol Clin Pharmacol 2015;31 (1):67-71.

[8] McDonnell JG, O'Donnell B, Curley G, et al. The analgesic efficacy of transversus abdominis plane block after abdominal surgery: a prospective randomized controlled trial. Anesth Analg 2007;104(1):193-7.

[9] Netter FH. Back and spinal cord. In: Netter FH. edr. Atlas of human anatomy summit. New Jersey, USA: The Ciba-Geigy Corporation 1989:145-55.
[10] Netter FH. Abdomen posterolateral abdominal wall. In: Netter FH. edr. Atlas of human anatomy summit. New Jersey, USA: The Ciba-Geigy Corporation 1989: 230-40.

[11] Lee TH, Barrington MJ, Tran TM, et al. Comparison of extent of sensory block following posterior and subcostal approaches to ultrasound-guided transversus abdominis plane block. Anaesth Intensive Care 2010;38(3):452-60.

[12] Atkinson RS, Rushman GB, Lee JA. A synopsis of anaesthesia. 10 th edn. Bristol: Wright 1987:637-40.

[13] Fuladi N, Deshmukh S, Bhur A. Comparative study of bupivacaine $0.25 \%$ versus ropivacaine $0.5 \%$ in transversus abdominis plane block for postoperative analgesia in lower abdominal surgeries: a randomised controlled trial. Journal of Evolution of Medical and Dental Sciences 2014;3(17):4569-76.

[14] Patil SS, Pawar SC, Divekar VM, et al. Transversus abdominis plane block for an emergency laparotomy in a high risk elderly patient. Indian Journal of Anaesthesia 2010:54(3):249-54. 